\title{
Stem cells and the modeling of the extracellular matrix-the library of cells needs suitable bookshelves
}

\author{
Stefan Lorenzl
}

Published online: 16 March 2010

(C) Springer-Verlag 2010

Amyotrophic lateral sclerosis, the most common form of motor neuron disease, is one of the most devastating diseases of mankind. The progressive loss of all efferent systems finally leads to the complete loss of the individual contact to the outside world. The progression of the disease is rapid and the majority of patients have a life expectancy of only 35 years. Despite considerable effort to investigate pathophysiologic mechanisms and develop different treatment strategies, no treatment has proven effective to slow disease progression. Beside multiple approaches using drug treatment, autologous stem cell therapy has been suggested as a promising therapeutic strategy since stem cells have the ability to differentiate into a variety of cell lines and could possibly restore damaged motor neurons or might even generate new ones. Stem cells, including the neuronal progenitor cells, are present in embryos but also in umbilical cord blood. In adults, these cells are present in bone marrow and in other organs where controlled self-renewal is needed [1]. A small portion of these cells is also present in the brain and might be used for memory functions [2].

Stem cell therapy might provide hope for patients with ALS in several ways. Ideally, stem cells could be induced to differentiate into lower motor neurons in order to replace those neurons that have died during the course of the disease. Moreover, they might rescue dying motor neurons from ultimate death by reconnecting them to denervated muscles. Even better, stem cells might be able to differentiate into upper motor neurons in the cortex and connect them to the lower motor neurons.

\section{S. Lorenzl $(\bowtie)$}

Department of Palliative Care,

Ludwig Maximilians University München,

Marchioninistrasse 15,

81377 München, Germany

e-mail: Stefan.Lorenzl@med.uni-muenchen.de
Are these expectations that stem cells will play a regenerative role in patients with ALS realistic? Currently, it seems unrealistic because of the complexity of the task; whereas transplantation of stem cells may be technically possible, trafficking and survival in the host tissue cannot be controlled sufficiently. A more reasonable scenario for stem cells in ALS treatment could be a supportive role in maintaining the viability of or extending the function of surviving motor neurons. Stem cells could be induced to differentiate into supporting cells, e.g., glia, or interneurons that produce supportive factors for motor neurons. Moreover, stem cells could be used as "Trojan horses" to transport growth factors or cytokines, or even deliver drugs to sites of degenerating neurons. Potential benefits of stem cell therapy in ALS have been demonstrated in experimental models by providing non-compromised supporting cells such as astrocytes, microglia or growth-factor-secreting cells, resulting in delayed onset of the disease and prolonged survival. In rodent models of acute or chronic motor neuron injury, neural stem cells implanted into the spinal cord have been shown to differentiate into motor neurons, with some evidence of axonal sprouting and formation of neuromuscular junctions with host muscle [3]. However, experimental results are controversial and transplantation studies in ALS mice showed that intrathecally implanted stem cells do not lead to a major therapeutic effect with regard to disease progression or survival time [4].

Especially the exploitation of bone marrow-derived mesenchymal stem cells (MSCs) appears to be an interesting therapeutic tool since these stem cells can be collected and manipulated easily. Moreover, MSCs appear capable to migrate easily through barriers of tissue layers, invade the central nervous system (CNS) and differentiate into neural cells. The privileged transdifferentiation of these cells is into glial cells, which has been described by Bossolasco 
[5]. Their glial properties render them a promising tool for a therapeutic approach since in ALS the cells around motor neurons deteriorate leaving the neuronal population without the necessary support of the extracellular matrix (ECM).

Matrix metalloproteinases (MMPs) are a family of structurally and functionally related zinc-dependent endopeptidases capable of degrading components of the ECM and basement membranes. MMP proteins are membrane bound and their inactive zymogen form requires proteolytic processing to release the catalytically active enzyme. MMP activity is regulated at multiple levels. The MMP genes are transcriptionally responsive to a wide variety of growth factors, cytokines, and reactive oxygen species. In the CNS, MMPs are synthesized by neurons, astrocytes, and microglia [6]. Accumulating evidence indicates that MMPs are involved in the pathogenesis of a number of CNS disorders including neuromuscular diseases [7]. Although the cause of ALS remains obscure, potential roles of MMPs have been extensively investigated.

In the developing CNS, MMPs are involved in neurogenesis, axonal guidance, and growth, myelinogenesis and angiogenesis [2]. In the adult CNS they play a role in remodeling of the extracellular matrix, cell migration, and survival, in synaptic plasticity with an impact on learning and memory function, myelin turnover, and angiogenesis MMP function is normally tightly regulated at all levels. Abnormal expression, the release of MMPs from dying cells and a lack of counter-regulation by the tissue inhibitors of MMPs (TIMPs) are implicated in CNS diseases. Moreover, at least some MMPs seem to have a direct neurotoxic effect like MMP-9 and MMP-3 [2] (Fig. 1).

\begin{tabular}{|c|c|}
\hline Developing CNS & Adult CNS \\
\hline $\begin{array}{l}\text { Neurogenesis } \\
\text { Axonal growth } \\
\text { Axonal guidance } \\
\text { Myelinogenesis }\end{array}$ & $\begin{array}{l}\text { Synaptic plasticity } \\
\text { Myelin turnover } \\
\text { Angiogenesis } \\
\text { Regulation of ECM }\end{array}$ \\
\hline CNS Repair & CNS Injury \\
\hline $\begin{array}{l}\text { Axonal growth } \\
\text { Axonal guidance } \\
\text { Remyelinisation } \\
\text { Angiogenesis }\end{array}$ & $\begin{array}{l}\text { Direct cytotoxicity } \\
\text { Myelin degradation } \\
\text { Axonal destruction } \\
\text { Processing of „death molecules“, e.g. FasL } \\
\text { Conversion of inflammatory molecules } \\
\text { Anoikis }\end{array}$ \\
\hline
\end{tabular}

Fig. 1 Illustrates the multiple roles of MMPs in the development and in the normal adult CNS. CNS diseases or injury leads to MMP dysfunction and consequently neuronal death. MMPs are involved in many pathways in CNS pathology which are also important in stem cell transplantation. Especially the changes of cell-matrix interaction and the direct cytotoxic effect of MMPs. However, the multiple roles of MMPs in CNS repair are important when considering stem cell transplantation (modified after reference 2)
It is well established that in ALS pathological changes not only occur in the nervous tissue but involve multiple areas, including the skin. Biochemical changes and disorganization of the extracellular matrix proteins of the skin have been described earlier [8] Fang et al. [9] found significant changes of MMP-9 in the cerebrospinal fluid and skin of patients with ALS and showed a correlation between MMP-9 levels and rapid disease progression. They suggested that the detrimental role of increased MMP-9 expression might be linked to collagen degradation in the skin, most prominently involving collagen type IV, which is the major component of the dermal ECM.

Kiaei et al. [10] identified MMP-9 to be the major factor contributing to motor neuron death in ALS. They showed increased MMP-9 immunoreactivity and MMP-9 activity in the spinal cord of G93A mice, a model for ALS carrying the G93A SOD mutation, as early as 40 days of age, and those levels continued to increase with age. They conclude that the creased MMP-9 activity at an early stage, prior to any symptoms, is suggestive for its important role in disease pathogenesis. They next cross-bred MMP-9 deficient mice with the G93A transgenic mouse, and found that MMP9 deficiency significantly attenuated neuronal loss and reduced neuronal TNF-alpha and FasL immunoreactivities in the lumbar spinal cord. Furthermore, the MMP-9-deficient offspring had a significantly longer live span than wild-type mice, confirming an earlier study in which treatment with a broad spectrum MMP inhibitor significantly prolonged life in G93A mice [11].

In this issue, Bossolasco et al. [12] tested the hypothesis that ALS is a systemic disease and that SCs, outside CNS tissue, are involved in the pathophysiology. To do that they examined several components of the hematopoietic/mesenchymal compartment of ALS patients and healthy donors by functional and molecular analysis. While Bossolasco et al. report normal hematopoietic and stromal cell properties of long-term bone marrow cultures of patients when compared with normal donors, they found disease-related differences in the levels of various MMPs in lysates from cultured mesenchymal stem cells of ALS patients and healthy donors, as well as delayed growth rate and reduced osteoblastic differentiative potential of patients' MSCs. The changes in MMP and TIMP expression comprise significant reduced levels of MMP-1, MMP-2, and MMP-3 as well as TIMP-2 in MSC of ALS patients. MMP-9 levels were found to be slightly elevated in single patients, but this finding did not reach a significant level. These ELISA data were confirmed by zymography, an elegant method to analyze the gelatinolytic activity of MMP-9 and MMP-2 (which are also called "gelatinases"). However, on the transcriptional level the authors found a significant upregulation of MMP-9 in MSC, which could imply a high rate of turnover of this enzyme. As mentioned above, MMPs 
not only have detrimental effects (e.g., myelin degradation, axonal destruction, direct cytotoxicity), but also play important roles in tissue remodeling. One might consider that the reduced levels of certain MMPs in MSCs might imply a failure of successful integration after transplantation. But we can only speculate which MMPs are helpful in this process.

Besides reduced levels of TIMP-2, the levels of TIMP-1 were also lowered in ALS patients which might be the result of an extended turnover of these proteins regulating excess MMP activity. TIMP proteins have multiple functions beside MMP counter-regulation, and the findings point toward an interesting role of TIMP regulation in ALS. Another counter-regulatory mechanism with regard to MMP activation might be exerted by collagen type IV which was also found to be elevated in the patients' MSC compartments. However, since the protein levels of MMPs have mainly been found reduced this remains speculation.

The study by Bossolasco et al. provides a lot of helpful information regarding stem cell therapy in ALS patients. Importantly, the changes in MSC indicate that there are multiple barriers at the enzymatic level which have to be considered using these cells for a therapeutic approach. Since we know that components of the ECM are altered in ALS patients, MSCs have the potential to enhance these alterations, and that might explain the failure of and conflicting results after bone-marrow derived stem cell transplantation in ALS animal models. The tissue microenvironment of the motor neurons plays an important role in cell survival and has only recently been recognized as a primary target of the pathophysiology. Since the ECM is a complex system regulating cell survival and function, disturbances of the balanced system lead to a loss of cellular homeostasis. Disruption of cell-matrix interaction has adverse effects on cell survival and can induce a specific type of apoptosis known as "anoikis" (Greek for homelessness) that is due to loss of cell adhesion or inappropriate cell adhesion. Transplanted cells are ultimately depending on the support by their environment including the extracellular matrix components. Dysregulation of the extracellular matrix components induced by transplanted stem cells contributes to tissue damage and even enhances the process of neurodegeneration.

The MMPs family of endoproteases comprises four types of enzyme classes, including the serine, cysteine, and aspartic proteases. These classes of enzymes are only barely investigated in motor neuron diseases and it is important to note, that these proteolytic systems are closely interacting in remodeling the extracellular environment. Therefore, only a detailed analysis of the cells and the surrounding tissue might explain the changes we observe in ALS.

However, the finding that ALS is associated with altered MMP expression in bone marrow mesenchymal stem cells is intriguing and of potential clinical relevance, and contributes to our understanding of stem cell transplantation in ALS patients. Furthermore, it demonstrates that ALS is a systemic disease.

We would certainly have to consider changes in the expression of certain genes of stem cells before transplanting them into humans. But we would also have to consider the components of the extracellular matrix, and the site where we transplant the cells. If the extracellular matrix is unable to provide enough support for survival, the transplanted cells will die because of insufficient cellular contact. The situation can be compared to a complex library where information can be retrieved only from well-placed books.

\section{References}

1. Silani V, Fogh I, Ratti A, Sassone J, Ciammola A, Cova L (2002) Stem cells in the treatment of amyotrophic lateral sclerosis. Amyotroph Lateral Scleros Other Motor Neuron Disord 3:173-181

2. Agrawal SM, Lau L, Yong VW (2008) MMPs in the central nervous system: where the good guys go bad. Semin Cell Dev Biol 19:42-51

3. Mazzini L, Vercelli A, Ferrero I, Mareschi K, Boido M, Servo S, Oggioni GD, Testa L, Monaco F, Fagioli F (2009) Stem cells in amyotrophic lateral sclerosis: state of the art. Expert Opin Biol Ther 9:1245-1258

4. Habisch HJ, Janowski M, Binder D, Kuzma-Kozakiewicz M, Widmann A, Habich A, Schwalenstöcker B, Hermann A, Brenner R, Lukomska B, Domanska-Janik K, Ludolph AC, Storch A (2007) Intrathecal application of neuroectodermally converted stem cells into a mouse model of ALS: limited intraparenchymal migration and survival narrows therapeutic effects. J Neural Transm 114:1395-1406

5. Bossolasco P, Cova L, Calzarossa C, Rimoldi SG, Borsotti C, Deliliers GL, Silani V, Soligo DPolli E (2005) Neuro-glial differentiation of human bone marrow stem cells in vitro. Exp Neurol 193:312-325

6. Gottschall PE, Deb S (1996) Regulation of matrix metallorpteinase expressions in astrocytes, microglia and neurons. Neuromodulation 3:69-75

7. Renaud S, Leppert D (2007) Matrix metalloproteinases in neuromuscular disease. Muscle Nerve 36:1-13

8. Kolde G, Bachus R, Ludolph AC (1996) Skin involvement in amyotrophic lateral sclerosis. Lancet 347:1226-1227

9. Fang L, Huber-Abel F, Teuchert M, Hendrich C, Dorst J, Schattauer D, Zettlmeissel H, Wlaschek M, Scharffetter-Kochanek K, Tumani H, Ludolph AC, Brettschneider J (2009) Linking neuron and skin: matrix metalloproteinases in amyotrophic lateral sclerosis (ALS). J Neurol Sci 285:62-66

10. Kiaei M, Kipiani K, Calingasan NY, Wille E, Chen J, Heissig B, Rafii S, Lorenzl S, Beal MF (2007) Matrix metalloproteinase-9 regulates TNF-alpha and FasL expression in neuronal, glial cells and its absence extends life in a transgenic mouse model of amyotrophic lateral sclerosis. Exp Neurol 205:74-81

11. Lorenzl S, Narr S, Angele B, Krell HW, Gregorio J, Kiaei M, Koedel U, Pfister HW, Beal MF (2006) The Matrix Metalloproteinases inhibitor Ro 26-2853 extends survival in transgenic ALS mice. Exp Neurol 200:166-171

12. Bossolasco P, Cova L, Calzarossa C, Servida F, Mencacci NE, Onida F, Polli E, Lambertenghi Deliliers G, Silani V. (2010) Metalloproteinase alterations in the bone marrow of ALS patients. J Mol Med. doi:10.1007/s00109-009-0584-7 